FERMILAB-Pub-87/184-E

[E-741/CDF]

\title{
The Design and Construction of the CDF Central Drift Tube Array*
}

S. Bhadra, S. Errede ${ }^{\star}$, L. Fishback, H. Keutelian, and P. Schlabach

Loomis Laboratory of Physics

The University of Illinois at Urbana-Champaign

1110 W. Green Street

Urbana, Illinois 61801 USA

July-August 1987

*Submitted to Nucl. Instrum. Methods A 



\title{
The Design and Construction of the CDF
}

\section{Central Drift Tube Array}

S. Bhadra, S. Errede*, L. Fishback, H. Keutelian, P. Schlabach

Loomis Laboratory of Physics

The University of Illinois at Urbana-Champaign

1110 W. Green Street

Urbana, Illinois, 61801 USA

August 4, 1987

\begin{abstract}
The Central Drift Tube (CDT) array is one of the charged particle tracking systems for the Collider Detector at Fermilab (CDF). The CDT array, which operates in the limited streamer mode, provides highaccuracy $r-\phi-z$ information at a radius of $1.4 \mathrm{~m}$ for tracking charged particles produced in the central region (pseudo-rapidity range $-1<\eta<1$ ) of $\sqrt{ } \mathrm{s}=1.8 \mathrm{TeV} \overline{\mathrm{p} p}$ collisions. Tracking of charged particles in the
\end{abstract}

-Alfred P. Sloan Research Fellow, 1985-89 
$r-z$ view of the CDT array is accomplished via charge division along the anode wires; drift-time measurements in three layers of the CDT array provide tracking information in the $r-\phi$ view. A novel design enabling rigid and precise mechanical support of $2,0163 \mathrm{~m}$ long, $12.7 \mathrm{~mm}$ diameter ultra-thin walled stainless steel drift tubes has been implemented for the CDF Central Drift Tube array. Details of the mechanical design and fabrication of the CDF Central Drift Tube array are described. Some preliminary performance results of the operation of the CDT array obtained from $\sqrt{\mathrm{s}}=1.8 \mathrm{TeV}$ p collisions are also discussed.

\section{Design Requirements}

The primary purpose of the Central Drift Tube (CDT) array for the Collider Detector at Fermilab (CDF) is to provide high-accuracy $r-\phi-z$ information at a radius of $1.4 \mathrm{~m}$ for tracking charged particles produced in the central region (pseudo-rapidity range $-1<\eta<1$ ) from $\sqrt{ } \mathrm{s}=1.8 \mathrm{TeV} \overline{\mathrm{p}} \mathrm{p}$ collisions. The CDT array is located on the outer cylindrical surface of the Central Tracking Chamber (CTC), and just inside the CDF solenoid magnet, which provides a uniform axial magnetic field of $B=1.5$ Tesla. Tracking of charged particles in the $r-z$ view of the CDT array is accomplished via charge division along the anode wires; drift-time measurements in three layers of the CDT array provide tracking information in the $r-\phi$ view. In off-line event track-reconstruction, overall CPU time is minimized by using track information from the CDT array in conjunction with track information from the axial and small angle $3^{\circ}$-degree 
stereo views in the CTC [1] and also using track information from the Vertex Time Projection Chamber (VTPC) [2] to obtain three-dimensional track information for charged particles produced in the central region of the CDF detector. Prompt hit information from the CDT array's RABBIT readout electronics, in the form of digital (TTL) signals and analog sums [3] can be used in conjunction with prompt signals from the Central Muon Chambers [4] and the Central Electromagnetic Calorimeter [5] to generate Level-II muon and electron triggers, respectively, for the central region of the CDF detector.

The Central Drift Tubes operate in the limited streamer mode to obtain the maximum possible $\mathrm{Z}$-resolution, $\sigma_{z} \leq 0.1 \% \mathrm{~L}$ via the method of charge division. In this mode of operation, amplification of the signals from the ends of each tube is not required and unwanted electronic noise, which would degrade the overall Z-resolution, is not introduced into the system. As a consequence, the design of the CDT array is simplified; the reliability and efficiency per channel is increased and the overall cost of the detector is reduced.

The number of charged particles observed in the CDT array for a typical $\sqrt{ } \mathrm{s}=1.8 \mathrm{TeV}$ pp event peaks at $\mathrm{N}_{\mathrm{ch}} \sim 16$. However, the fluctuations on $\mathrm{N}_{\mathrm{ch}}$ are such that the tail of this distribution extends out to beyond 100 charged particles per event. In order to minimize the probability of charge division information being corrupted by two or more tracks passing through the same drift tube, fine segmentation in $r-\phi$ is needed. Guided by several Monte Carlo simulation studies of minimum bias and high- $P_{t}$ jet events from $\sqrt{ } \mathrm{s}=$ 
$1.8 \mathrm{TeV}$ p p collisions [6], the optimum, fundamental cell size for the CDT array was determined to be on the order of $1 \mathrm{~cm}^{2}$ area. This cell size was also commensurate with considerations of the mechanical, electrical, triggering, offline track reconstruction aspects and overall cost for the array. The overall length the CDT array was constrained to be on the order of $3 \mathrm{~m}$ by the length of the CTC. The radial thickness of the CDT array was constrained by the dimensions of the outer radius of the CTC and the bore of the solenoid magnet to be $\Delta \mathrm{R}<3.8 \mathrm{~cm}$.

In order to achieve a high overall track efficiency, especially important for radial (i.e. high- $P_{t}$ ) tracks, and to have a complete, redundant, self-consistent and independent means of understanding calibration constants, potential detector biases, systematic effects, etc., the design of the Central Drift Tube array incorporated three layers of drift tubes instead of only two. Due to budgetary limitations, only half of the total number of required RABBIT readout channels of $\mathrm{ADC} / \mathrm{TDC}$ electronics could be implemented for the first data-taking run of CDF. In order to accomodate this constraint, pairs of drift tubes were ganged together in the same layer, separated by eight tubes. The Monte Carlo simulation studies of the CDT array indicated that this choice was a reasonable and efficient drift tube ganging configuration.

To maintain the intrinsically high Z-resolution of drift tubes operated in the limited streamer mode, it is extremely important to have good noise immunity and the ability to calibrate each tube insitu. Great care was taken to ensure 
proper electrical grounding and full $4 \pi$ enclosed of shielding of the signals from the CDT array. The routing of all signal, $\mathrm{HV}$ and intrumentation cables going to and from the array was also carefully considered in order to minimize induced pickup of electrical noise from $(\partial \overrightarrow{\mathrm{B}} / \partial t \cdot \overrightarrow{\mathrm{A}})$ effects. Weak $\mathrm{Fe}^{55}$ sources positioned at precise locations near the ends of each drift tube provide a means for Z-calibration and also a means for monitoring the daily operational performance of the CDT array. An electronic pulsing system for charge injection enables the calibration and monitoring of the CDT array's signal cables and RABBIT ADC/TDC electronics channels. The gas input/output pressures and ambient temperature of the CDT array are monitored via 24 channels of solidstate pressure transducers and 72 channels of temperature sensors strategically located around the detector.

The CDT array is located inside the superconducting solenoid coil and is in proximity to the inner cryostat wall of the coil. To compensate for the $\sim 30 \mathrm{Watt} / \mathrm{m}^{2}$ heat leak into the solenoid through the cryostat wall, the outer surface of the CDT array is inlaid with a compact, twisted-pair nichrome wire "heater" system, which maintains a constant, uniform temperature of $20 \pm 2{ }^{\circ} \mathrm{C}$ around the array. The CDT heater circuitry also locally compensates for heat losses due to the water cooling of the CTC preamplfiers, located on the CTC endplates.

The net thickness and type of materials used in the fabrication of CDT array were also carefully considered, to minimize the effects of secondary particle inter- 
actions and multiple coulomb scattering, etc. on other CDF detectors external to the CDT array, most notably the Central EM and Hadronic Calorimeters, while still maintaining structural rigidity and integrity of the CDT array.

Finally, since the CD' array is physically attached to the CTC, the design of the CDT array is such that it is electrically isolated from the CTC to suppress unwanted electrical noise and eliminate ground loops.

\section{The Design of the CDF Central Drift Tube Array}

In the early stages of the design of the Central Drift Tube array, much time and effort was spent considering various possible cell geometries. Several open cell designs were considered, but were discarded because of design complexities, mechanical tolerance and performance considerations, and concerns over potential problems resulting from anode wire breakage. Individual closed-cell geometries in a wide variety of shapes and sizes, from triangles to rectangles and squares, to hexagons and circular cells, to aluminized mylar soda-straw tubes were all considered. The various (simultaneous) concerns here were associated with the packing fractions, cell sizes, minimizing overall material thickness, minimizing wall thicknesses and effective dead space between neighboring cells, maximizing overall mechanical and structural rigidity, electric field nonuniformities and $(\overrightarrow{\mathrm{E}} \times \overrightarrow{\mathrm{B}})$ effects, uniform gas distribution, gas flow and pressure, potential gas sealing problems, providing a means for Z-calibration of each tube via weak radioactive sources, the relative difficulties associated with machin- 
ing and fabrication of the various needed components for the CDT array, and the overall machining, fabrication and labor costs associated with each of the candidate closed-cell designs for the CD'T array.

After much consideration, a closed-cell design consisting of cylindricallyshaped hard-drawn thin-walled metal tubes was decided upon as the best overall geometry for use in the CDT array. Explicit measurements and calculations of the rigidity of individual tubes and clusters of tubes bonded together with various adhesives such as structural epoxies, cyano-acrylates, etc. were carried out for several types and wall-thicknesses of aluminum, brass and stainless steel tubes. In-depth studies of the bonding strengths of drift tubes glued together with various adhesives were carried out. Studies of the effect of different surface preparations on tube bonding strength were also done [7].

Several computer-aided design (CAD) programs were written to help obtain the optimum design configuration of tubes, optimum tube diameter and packing fraction. Guided by the Monte Carlo simulation studies of minimum bias and high- $P_{t}$ jet events from $\sqrt{ } \mathrm{s}=1.8 \mathrm{TeV} \bar{p}$ collisions [6], the final design of the CDT array consisted of $2,016 \quad 12.7 \mathrm{~mm}$ diameter tubes, $3 \mathrm{~m}$ in length, arranged in three layers of 672 tubes per layer. To enhance structural rigidity and to ease fabrication and handling, the CDT array was made in twelve interlocking $30^{\circ}$ sectors. Each drift tube sector consists of 168 drift tubes organized in three close-packed layers of 56 drift tubes per layer. As shown in Fig. 1, the drift tubes in the middle layer of the CDT array are offset in $\phi$ by 0.5 tube diameter 
relative to the inner layer of drift tubes. The drift tubes in the outermost layer of the CDT array are offset in $\phi$ by 0.1 tube diameter relative to the inner layer of drift tubes, thereby guaranteeing that at least two drift tubes will always be traversed for a radial track of infinite momentum. The design of a $30^{\circ} \mathrm{CDT}$ sector is shown in Fig. 2.

The use of the aluminum sector ribs is fundamental to the design of the CDT array and is one of the key factors in achieving the structural rigidity of the array and in maintaining precision alignment of drift tubes along their entire length. Each sector of drift tubes uses six precision NCM-machined 6061 T-6 alumimum "swiss-cheese" sector ribs. The aluminum sector ribs have 168 precision-milled drift tube alignment holes (oversized $25 \mu \mathrm{m}$ ), which are used to maintain tube alignment over the $3 \mathrm{~m}$ tube length at $\sim 60 \mathrm{~cm}$ intervals. The fractional dead space associated with each layer of the CDT array (as seen by a radial track) due to the finite drift tube wall thickness and gaps between adjacent drift tubes is $5.2 \%, 5.9 \%$, and $6.7 \%$ for the inner, middle and outermost layers of the CDT array, respectively.

Several $30^{\circ}$ prototype sectors of aluminum tubes were built to evaluate their mechanical rigidity and other structural properties of the CDT array, and also for the purposes of developing tube bonding techniques and to evaluate tube bonding agents. These mechanical studies also enabled quantitative comparison of explicit measurements with theoretical calculations of the mechanical properties of the prototype drift tube sectors, such as the amount of sag. The 
agreement between calculated and measured properties of these prototype sectors of drift tubes was very good. We also learned from these mechanical studies that drift tube sectors built without the use of aluminum sector ribs were not nearly as structurally robust as ones built with the use of the aluminum sector ribs. In the former case, precision alignment of drift tubes in three dimensions was much more difficult to maintain and fabrication of drift tube sectors was also considerably more complicated and lengthy.

Several prototype aluminum, brass and stainless steel drift tubes were made. We evaluated their performance characteristics and studied their operation in the limited streamer mode [8]. Three different anode wire diameters and two types of resistive stainless steel anode wire were also tested. Drift tubes made out of aluminum or brass were initially favored because the cost per unit length of material was considerably less than that of stainless steel tubes. Aluminum tubes were also favored because the overall thickness of the CDT array, in terms of radiation lengths of material, would have been the smallest, at $\sim 3 \% \chi_{\circ}$ for a radial track vs. $\sim 10 \% \chi_{\circ}$ for an array composed of stainless steel tubes. However, we discovered that aluminum drift tubes operated in the limited streamer mode inevitably developed "hot spots" along the wire due to glow discharge from the buildup of ionic charge on the aluminum oxide layer on the inner wall of the aluminum tube, and subsequent emission of Malter electrons. We found that this unpleasant effect could only be eliminated by plating aluminum tubes with a non-surface-oxidizing metal, such as nickel, which is a costly process. 
Aluminum is also a relatively soft metal, easily scored and/or scratched, usually leaving behind metal burrs (which also form "hot spots" in the limited streamer mode if not removed). We also observed a relatively large variation in wallthickness and tube non-uniformity in the sample of $\sim 500$ aluminum tubes used for the mechanical studies. We therefore decided against the use of aluminum tubes for the CDT array. Brass tubes, while being extremely cheap and uniform in wall-thickness were found to have unacceptable variations in tube roundness. Brass is structurally much weaker than either aluminum or stainless steel. Drift tubes made out of brass were both predicted and measured to sag a factor of $\sim$ $2 x$ more than either aluminum or stainless steel tubes. Brass is also chemically reactive, and tarnishes over a period of time in air. We were concerned with potential long-term effect(s) of trace amounts of moisture and other impurities contained in the alcohol in the argon-ethane-alcohol gas mixture reacting with the inner surface of the brass tubes. For these reasons (and others) the use of brass drift tubes for the CDT array was also discarded.

Stainless steel tubes, while more costly and of a higher Z-material than aluminum, were found to be mechanically advantageous to that of either aluminum or brass tubes. We were able to obtain hard-drawn non-magnetic 304 stainless steel tubes which had extremely uniform, $12.71 \pm 0.01 \mathrm{~mm}$ tube o.d. and $200 \pm 10 \mu \mathrm{m}$ wall-thickness which were also extremely straight [9]. Stainless steel tubes, while weighing $3 x$ more than aluminum tubes, sag the same amount as aluminum tubes, because the ratio of the mass density/Young's modulus is 
nearly the same for these two materials. Stainless steel is also much less chemically reactive than either aluminum or brass, has no significant oxide layer and also does not score, scratch or dent nearly as easily as aluminum.

The $3 \mathrm{~m}$ length of each drift tube makes it necessary to provide a means of support for the anode wire mid-length along the tube to prevent wire instability at high voltage. The design of the drift tube delrin wire support is shown in Fig. 3. The $1.6 \mathrm{~mm}$ diameter brass insert at the center of the wire support reduces the value of the electric field by $\sim 1$ order of magnitude at the interface between the delrin and the anode wire and prevents HV discharge from occurring at this point. The central "hub" of the delrin wire support is also deliberately shaped to inhibit HV discharge. Three holes in the wire support allow gas to flow through the tube. The shape of the outer rim of the wire support ensures reliable and precise positioning of the support and the anode wire inside the tube by using a specially developed insertion tool. Due to the presence of the wire support, the drift tube is inefficient in this region. Wire supports for different drift tubes in a CDT sector are staggered in a well-defined manner using 8 distinct wire support locations, so that charged particles traversing the array in the vicinity of the wire supports are affected by at most one wire support [10].

The design of the CDT delrin endpin/feedthru is shown in Fig. 4. The brass crimp tube pin assembly consists of an "inverse hour-glass" shaped brass insert crimped to the end of the brass crimp tube, which defines the point of electrical contact between the resistive anode wire and the brass crimp tube pin 
to an accuracy of $\pm 100 \mu \mathrm{m}$. The design of the brass insert is also such that it enables easy, bi-directional passage of the anode wire through the $75 \mu \mathrm{m}$ diameter central hole of the brass crimp tube insert. This important feature considerably eased the drift tube wire stringing process. Three holes in the delrin endpin/feedthru allow gas to flow freely into and out of the tube. A Viton O-ring provides gas sealing for each drift tube [11].

The design of the end assemblies for the CDT array is rather complicated. This structure simultaneously performs many functions. The end assemblies provide rigid mechanical support and also a means for attaching each CDT $30^{\circ}$ sector to the CTC. The end assemblies also provide a means for supplying HV to each drift tube anode wire and contain the blocking capacitors needed for extracting the signals from the ends of each drift tube. In addition, a means for distributing gas to each drift tube is provided for in the design of the end assemblies. In order to minimize electronic cross-talk between neighboring drift tubes, and to provide good $\mathrm{rf}$ shielding in this region of the array, each drift tube has its own individual and extremely compact pair of "shotgun shells". Each shotgun shell, $101.6 \mathrm{~mm}$ in length, was made of $150 \mu \mathrm{m}$ wall-thickness hard-drawn cartridge brass. The shotgun shells have the same outer diameter as the stainless steel drift tubes and contain all the components necessary for the proper functioning of the drift tube. Each shotgun shell contained a 0.033 $\mu \mathrm{F}$ HV blocking capacitor [12], two 1N914B clamping diodes, a $1.0 \mathrm{Meg}-\mathrm{Ohm}$ HV current-limiting resistor, a 1.0 Meg-Ohm load resistor, teflon tubing for 
gas flow, delrin and brass parts for internal position registration of parts, small p.c. boards, a conductive rubber connection, signal and BV cables, and an insulating mylar film barrier. A detailed view of one of the brass shotgun shell end-assemblies is shown in Fig. 5.

For the sake of modularity and concerns about potential problems associated with wire replacement, etc., shotgun shells are grouped together in units of 24 , in three rows of 8 tubes. Two precision NCM-machined 6061 T-6 aluminum plates, spaced apart and held together by 8 precision-ground $1.6 \mathrm{~mm}$ diameter brass standoffs and 0-80 stainless steel screws, hold the 24 brass shotgun shells in precise alignment. Fig. 6 shows the details associated with a complete endassembly unit, termed a "HV pod". Each CDT $30^{\circ}$ sector has $7 \mathrm{HV}$ pods attached to each end of the sector for a total of $84 \mathrm{HV}$ pods in the array. Each HV pod bolts onto a CDT sector via 8 precision-ground $1.6 \mathrm{~mm}$ diameter brass standoffs.

From studies with a 13-tube drift tube prototype "mini-sector", we learned that in order for all drift tubes to operate with identical performance characteristics, such as equal pulse heights and drift tube efficiencies, it was very important for all tubes to have the same gas pressure. The formation of limited streamer pulses depends critically on both the electric field intensity and gas density. Therefore for each CDT sector, gas is distributed in parallel and at equal pressure to each drift tube via common input and output gas manifolds. The CDT gas manifolds are made of two hard-coat anodized, NCM-machined 
tool i jig-plate aluminum pieces laminated together. They are bolted onto the rear surface of the HV pods with $56 \quad 0-80$ stainless steel screws. Gas sealing is provided by 168 "quad" Viton O-ring seals seated between precision delrin gas standoffs on the rear surface of the HV pods and the front surface of the gas manifold.

High voltage is distributed to individual drift tubes via miniature HV coax cables and HV connectors [13] to delrin HV distribution boxes mounted on the rear surface of the gas manifolds of each of the 12 CDT sectors. There is one delrin HV distribution box per HV pod. All drift tubes in the array operate at the same potential of $\mathrm{HV}=2770 \mathrm{~V}$. The streamer pulse signals from the drift tubes in a HV pod exit from the rear of each shotgun shell via $50 \Omega$ micro-coax cables. The 24 micro-coax signal cables are soldered to printed circuit boards which have 60-pin miniature surface-mount connectors attached to them [14]. The p.c. board signal cable assemblies are bolted to the rear surface of the CDT gas manifolds using the same 0-80 stainless steel screws that are used to attach the CDT gas manifold to the HV pods. 26-conductor $50 \Omega$ micro-coax flat cables [15], $15 \mathrm{~m}$ in length, transport the signals from the CDT array to the RABBIT readout electronics located on the endwalls of the CDF Central Detector.

\section{Fabrication of the CDF Central Drift Tube Array}

For the fabrication of the Central Drift Tube array, personnel working on 
the project were divided into two groups. One team concentrated on the various tasks associated with the fabrication of individual drift tubes and CDT sector fabrication, the second group dealt with the many-fold tasks associated with the fabrication of the end assemblies for the CDT array. All components used in the array were subjected to rigorous inspection and testing at every stage of the fabrication process. Fabrication algorithms for each operation were documented so that all components were fabricated, inspected and tested by different personnel in the same manner. Data associated with measurements of critical parameters, such as the length of each drift tube, $\mathrm{Fe}^{55}$ source locations, anode wire resistances, wire tension measurements, etc. were stored on disk in a computer fabrication database. This information could be easily retrieved and analyzed at any time during the fabrication of the CDT array, providing instantaneous and invaluable feedback associated with quality control, production rates, etc.

Drift tube fabrication began with the sanding of the entire outer surface of the as-yet uncut stainless steel tubes using 400-grit emery paper. A sanding jig supported the lower cylindrical portion along the entire length of the tube to prevent deformation of the tube during the sanding process. From explicit tests we learned that the bond strength between adjacent tubes improved significantly if the bonding surface was slightly abraded and then thoroughly cleaned [7]. The bond strength between drift tubes was also found, interestingly enough, to "peak" at this \# grit emery paper. After sanding, tubes were washed in a bath of hot water $+1 \%$ Alconox solution, rinsed in a de-ionized water bath and then 
air-dried.

The stainless steel tubes were then cut to the precise length of $L=3048.000 \pm$ $0.050 \mathrm{~mm}$ using a fine ceramic saw blade lubricated with de-ionized water [16] The ceramic saw blade was mounted on a tool-post grinder motor which rotated at $10,000 \mathrm{rpm}$. A precision jig held each tube as it was cut. Care was taken to maintain constant, stable room temperature when the stainless steel tubes were being cut. The ends of each cut tube were de-burred and lightly filed smooth. After the cut tubes were allowed a few minutes to attain thermal equilibrium with the ambient temperature of the room, their lengths were measured to an accuracy of $\pm 10 \mu \mathrm{m}$. A precision length, $12.7 \mathrm{~mm}$ diameter solid 304 stainless steel rod was used as a reference standard to set up the ceramic saw cutting jig and drift tube length measuring jig at the beginning of each tube cutting session. These set-ups were also re-checked during and at the end of the drift tube cutting sessions. As each tube was cut and measured, a unique serial number was engraved on one end of the tube. The deviation of the measured drift tube lengths from the nominal design length for 2,256 cut tubes is shown in Fig. 7.

After the tubes were cut to length, $300 \pm 25 \mu \mathrm{m}$ diameter $\mathrm{Fe}^{35}$ source holes were electro-discharge machined (EDM-ed) through the wall of each tube at a distance of $152.40 \pm 0.15 \mathrm{~mm}$ in from the tube ends. The EDM-ing process, done with the use of de-ionized water, left a burr-free hole of uniform size in the wall of the tube. Fig. 8 shows the deviation of the measured $\mathrm{Fe}^{55}$ source hole 
locations from the nominal design location for 2,226 EDM-ed tubes.

In order for the drift tubes to operate reliably and to prevent formation of "hot spots", it is extremely important that the inside of each tube be absolutely clean and have no surface defects whatsoever. Therefore, all stainless steel tubes were thoroughly washed inside and out in a bath of hot water $+1 \%$ Alconox (by personnel using powder-free surgical gloves) and by using many small, lint-free sponges pushed through the inner bore of each drift tube using jets of hot water and Alconox until they were clean. Tubes were immediately rinsed in a bath of hot water, where more sponges were used to remove any remaining traces of Alconox solution from the inner bore of the tube. Tubes were further rinsed in a bath containing a non-flammable mixture of de-ionized water and reagent-grade ethanol. Tubes were then removed from this bath, stacked up on a draining rack and allowed to drain for a short period of time. Finally, the tubes were dried using a ultra-clean, ultra-dry source of compressed air [17]. The inner bore of each tube was immediately inspected for any traces of dirt and or flaws on the inner walls of the drift tube. Tubes which passed this inspection had their ends and $\mathrm{Fe}^{55}$ source holes temporarily sealed off using kapton tape [18]. The fraction of tubes which did not pass this visual inspection $(\sim 10 \%)$ were sent back to the beginning of the tube washing process. The few tubes which had flaws on the inner wall surface were discarded, and not used in the CDT array. Once drift tubes passed visual inspection, they were handled with clean, non-powdered surgical gloves until $30^{\circ}$ sector fabrication was completed. 
In a clean-room environment, the $50 \mu \mathrm{m}$ diameter stainless steel Stablohm800 anode wires [19] were strung down individual tubes using specially designed stringing jigs. The anode wire was first inserted through the brass crimp tube of one of the delrin endpin/feedthru's. The anode wire was attached to a small piece of tefion-covered soft iron and then "fished" through the drift tube using a small bar magnet. The wire support was back-inserted into the drift tube with the anode wire strung through the tube using one of four specially-designed wire support insertion tools to position the wire support precisely at one of four possible locations. The wire support was then crimped in place via threepoint compression at the outer surface of the drift tube. The wire support insertion tool was then removed from the drift tube, fresh wire pulled through, and the second delrin endpin/feedthru installed. The delrin endpin/feedthru's were then glued into the drift tube using 5-minute epoxy, a 22 AWG soft copper slug inserted into the crimp tube of the first endpin/feedthru and then crimped. The anode wire was then weighted with a tension of $T=100.0 \mathrm{gm}$ weight plus a $8.0 \mathrm{gm}$ kapton tape leader attached to the anode wire, and the second endpin/feedthru and copper slug crimped. With this technique, we experienced very few problems with wire slippage and/or wire breakage during the drift tube wire stringing process. Wire tension uniformity and reproducability were extremely good.

After drift tubes were strung, their lengths and anode wire resistances were measured. Anode wire resistances were nominally $\sim 1988 \pm 3 \Omega$. Drift tube wire 
tensions were measured using a computer-controlled wire tension measurement system [20]. Drift tubes were re-strung if the delrin endpin/feedthru's were not pushed fully into the drift tube, or if wire tension was less than $100.0 \mathrm{gms}$, or more than 120.0 gms. Less than $1.0 \%$ of all 2,226 drift tubes made had to be re-strung due to wire tension or tube length problems. Fig. 9 shows the wire tension distribution for 2,226 strung drift tubes, measured with the computer-controlled wire tension measurement system. Tubes which passed the wire tension measurement test were then BV tested in air to 2900 volts. Only three out of the 2,226 strung tubes failed this test, which consisted of visually monitoring the "signals" from each end of the drift tube on an oscilloscope and scaling these signals, which were discriminated at the $5 \mathrm{mV}$ signal level. Tubes with a singles counting rate greater than $2.5 \mathrm{~Hz}$ at $2900 \mathrm{v}$ in air or a discernable trace on the most sensitive scale of the oscillosope were rejected.

In order to obtain Z-calibrations for each tube, weak $(10.0 \pm 1.0 \mu \mathrm{Ci}) \mathrm{Fe}^{55}$ sources irradiate the anode wire of each drift tube through the $300 \pm 25 \mu \mathrm{m}$ EDMed holes near the ends of each drift tube. The copper-backed stainless steel $\mathrm{Fe}^{55}$ foil sources were cut into individual $2.54 \mathrm{~mm} \times 2.54 \mathrm{~mm}$ squares, of $200 \mu \mathrm{m}$ total thickness [21]. Due to the compactness of the CDT array and size of each drift tube, the use of these sources was ideal because of their extremely low profile. The activity over the total quantity of source material was uniform to $\pm 10 \%$, due to the unique method used by the vendor to "activate" the stainless steel foil. Each $\mathrm{Fe}^{55}$ source was attached to a $6.35 \mathrm{~mm}$ diameter $50 \mu \mathrm{m}$ thick kapton tape 
"dot" from the rear (copper) surface of the $\mathrm{Fe}^{55}$ source. Its activity was then explicitly measured using a short, $30 \mathrm{~cm}$ long drift tube dedicated specifically for the task of $\mathrm{Fe}^{55}$ source testing. In order to obtain uniform statistics during CDT Z-calibration data-taking runs, $\mathrm{Fe}^{35}$ sources which had singles counting rates more than $3 \sigma$ from the mean were not used. Because of the extreme sensitivity of the drift tubes to the presence of even single drift electrons inside the gas volume of the tube, we discovered that unless the front surface of each $\mathrm{Fe}^{55}$ source was covered (by even a few $\mu \mathrm{m}$ thick layer of tape), the singles counting rate from a bare, unshielded $\mathrm{Fe}^{55}$ source was a factor of $\sim 20 \mathrm{x}$ greater than that for a "shielded" source, due to the emission of Auger electrons. Thus, the front faces of all $\mathrm{Fe}^{55}$ sources were also covered with a $50 \mu \mathrm{m}$ thick, $2.6 \mathrm{~mm}$ x $2.6 \mathrm{~mm}$ square kapton tape "dot". The addition of this protective layer of kapton tape totally eliminated that portion of the singles counting rate due to the emission of Auger electrons from the $\mathrm{Fe}^{55}$ sources, and resulted in a $\sim 15 \%$ reduction in the singles counting rate due to the $5.9 \mathrm{KeV} \mathrm{X}$-rays. The strength of the $\mathrm{Fe}^{55}$ sources, along with the $300 \mu \mathrm{m}$ diameter of the EDM-ed $\mathrm{Fe}^{55}$ source holes, were deliberately chosen so that the singles counting rate from each $\mathrm{Fe}^{55}$ source was equal to the singles counting rate due to the ambient cosmic ray and natural radioactivity background, $\sim 15 \mathrm{~Hz}$ integrated over the entire length of the drift tube.

Fabrication of $\mathrm{CDT} 30^{\circ}$ sectors was accomplished using an extremely robust steel jig supported on two optically levelled granite tables. The sector fabrication 
jig consisted of two precisely aligned steel side-rails which held in precise location (via hardened steel dowel pins) six NCM-machined 6061 T- 6 aluminum "stock" assemblies which captured and precisely positioned each of the six "swiss-cheese" 6061 T-6 aluminum CDT sector ribs (again using hardened steel dowel pins). Two $5 \mathrm{~cm}$ thick precision aluminum tool \& jig-plate reference endplates, flat to $\pm 25 \mu \mathrm{m}$, were used to precisely align the Z-position of the drift tubes relative to each other. The alignment and registration of the sector jig was carefully checked prior to the fabrication of each CDT sector. The aluminum ribs, sector "stocks" and reference endplates were thoroughly cleaned prior to fabrication of each CDT sector. Key surfaces were pre-coated with a non-stick tefion compound to prevent gluing the CDT sector into the sector jig.

A sector map was used in the fabrication of each drift tube $30^{\circ}$ sector. This map specified the allowed locations of each of the four types of drift tubes, as defined by the positioning of the delrin wire support in the tube at the time the tube was strung. Drift tubes were first wiped down with ethanol, the serial number and type of drift tube checked and then simultaneously transcribed on the sector map and in a special logbook, as well as into the CDT fabrication data base. A gauging block was used to check the outer diameter of the ends of each tube. The protective kapton tape over the $\mathrm{Fe}^{55}$ source holes was removed. The drift tubes were then inserted one at a time into the holes of the aluminum sector ribs, and pushed flush against the reference endplate at the far end of the sector jig. After each tube was inserted into the sector, $\mathrm{Fe}^{55}$ sources were 
installed on each tube, and documented in another logbook. Adjacent pairs of drift tubes were rotated such that the $\mathrm{Fe}^{55}$ sources from the drift tube pair faced each other, to prevent being scraped off by subsequent installation of the remaining layers of drift tubes. This rotation of adjacent pairs of drift tubes also facilitated potting the area in the neighborhood of each of the $\mathrm{Fe}^{53}$ sources with structural epoxy to guarantee gas sealing. As a precaution, the wire resistance of each installed tube was also checked for continuity and for shorts to ground. When installation of the innermost layer of 56 drift tubes was complete, the nearside reference endplate was dowel pinned in place. The drift tubes were then "tack-bonded" to each of the aluminum ribs using a cyano-acrylate glue, which easily penetrated the small gaps between the aluminum ribs and the drift tubes. Dexter-Hysol 3X-Gray structural epoxy was then used to bond adjacent drift tubes together [22]. This epoxy was chosen for use in the fabrication of the CDT sectors because of its vastly superior bonding strength [7]. However, at room temperature, this epoxy is quite viscous. Considerable effort was made to obtain a reasonable technique for mixing and applying this glue. It was discovered that by simply elevating the temperature of the two components of the epoxy before mixing to $\sim 40^{\circ} \mathrm{C}$, a considerable reduction in the viscosity of two components occurred. The epoxy could then be mixed much more readily, thoroughly and rapidly. The mixed epoxy, made up in $\sim 200$ cc quantities, was then transferred to several $50 \mathrm{cc}$ disposable syringes with large-bore needles, re-heated to $\sim 65^{\circ} \mathrm{C}$ with a portable heat gun and then immediately applied by hand. Adjacent drift 
tubes were bonded together along their entire length. From explicit tests of this gluing technique, no significant reduction in the bonding strength of the epoxy was observed. The epoxy cooled rapidly to room temperature shortly after it was applied. When the epoxy bonding of the first layer of tubes was complete, the near-side reference endplate was removed. As each tube on the second layer was installed in the sector, the $\mathrm{Fe}^{53}$ sources were installed, anode wire continuity was checked, etc. Each drift tube was epoxy bonded to its neighbor in the second layer, as well as to one of its neighbors in the first row. Approximately one hour after installation of the second row of drift tubes began, the near-side reference endplate was re-installed, and these tubes were cyano-acrylated to each of the six aluminum ribs. The near-side reference endplate was then removed, and the process repeated until all drift tubes had been installed and epoxy bonded into place. The aluminum sector ribs located at either end of the CDT sector had 56 0-80 "anchors" which were also epoxy bonded in place. These anchors were used to provide additional structural strength to these end ribs, to which the CD'T HV pods were eventually attached. The sector fabrication process took an average of $\sim 12$ hours per CDT sector to complete with six personnel. Once the process had begun, it could not be easily interrupted. Approximately four liters of epoxy and $\sim 10 \mathrm{cc}$ of cyano-acrylate were used in the fabrication of one CDT $30^{\circ}$ sector. The newly-fabricated CDT sector remained in the sector jig for a period of 24 hours to allow the epoxy to fully cure. The CDT sector was then removed from the sector jig, flipped over and transferred to another 
pair of granite tables where it was placed on a smaller support jig, such that the innermost layer of drift tubes could also be epoxy bonded from this side. The sector jig was re-cleaned, the alignment re-checked, and then set up for the fabrication of another CDT sector. A total of $13 \mathrm{CDT}$ sectors were made. The first CDT sector made (\#00), was designated as the "official spare".

Fabrication of the brass shotgun shell end assemblies began by cutting 12.7 mm diameter brass tubing [23] with the ceramic saw to the precise length of $101.600 \pm 0.050 \mathrm{~mm}$ to make shotgun shell tubes. The ends of the shotgun shells were de-burred and lightly filed smooth. The length of each shotgun shell was measured to an accuracy of $\pm 10 \mu \mathrm{m}$, and a unique serial number engraved on its side. Each shotgun shell was then thoroughly scrubbed and cleaned with a mildly abrasive cleanser and water solution to remove the oxide layer, rinsed in de-ionized water and then air-dried. After drying, shotgun shells were stored in zip-lock plastic bags for later use.

The electrical and mechanical components internal to each shotgun shell were assembled on specially designed jigs to maintain precise location and orientation of each of the individual components. Another jig was used to precisely position the assembled internal components inside the brass shotgun shell. A small circular plated p.c. board at the rear of the shotgun shell was then soldered to the shotgun shell for grounding and if shielding purposes. Each shotgun shell was then tested for proper functioning signal-wise, using a fast pulse generator and an oscilloscope. 
The shotgun shell end assemblies were then potted with vacuum-degassified RTV-11 (specially chosen for its high voltage properties) [24] by injecting the RTV-11 into each shotgun shell with a high pressure air gun and with the use of another specially developed miniature positioning jig. The purpose of this jig was to precisely locate the internal delrin endplug with respect to the front end edge of the shotgun shell. The RTV-11 and the $125 \mu \mathrm{m}$ - thick insulating layer of mylar film, wrapped around the assembled components internal to the shotgun shell were crucial for the prevention of $\mathrm{HV}$ discharge inside the shotgun shell. When the RTV-11 had cured, the positioning jig was removed from each shotgun shell. The relative positioning of the delrin endplug to the front end of the shotgun shell was then checked with a depth gauge. Each shotgun shell was HV tested to 3000 volts and then gas leak tested at high pressure. The gas flow impedance of each shotgun shell was also checked for constriction or blockage of the teflon gas lines. Shotgun shells failing any of the of the above tests were either repaired, or taken apart and their usable parts recycled.

The CDT array's $168 \mathrm{HV}$ pods were made using groups of 24 shotgun shells. The 24 micro-coax signal cables associated with the shotgun shells were soldered onto a p.c. board to which the CDT array's signal cables would later be attached. The completed HV pods were then checked for HV and signal continuity. They were also signal-tested again using a fast pulse generator and an oscilloscope.

The HV pods were then installed on the ends of the CDT $30^{\circ}$ sectors. Spe- 
cially developed HV pod insertion tooling was required for this purpase. A zerostress electrical connection between each of the drift tube endpin/feedthrus and its corresponding shotgun shell end assemblies was made via a $12.7 \mathrm{~mm}$ long, $6.35 \mathrm{~mm}$ diameter conducting rubber segment [25]. The anode wire continuity of each drift tube was checked before and after HV pods were installed. "Quad" Viton O-ring gas seals [26] for each shotgun shell and $200 \mu \mathrm{m}$ thick insulating mylar retaining plates (used to position and hold the signal and $\mathrm{HV}$ cables in place) were then installed on the rear portion of each BV pod. Solid state pressure transducers [27] were installed on each of the gas manifolds, and 6 temperature sensors [28] were installed on the outer surface of each CDT sector. The aluminum gas manifold assemblies were then installed onto the ends of the HV pods, the final HV distribution cable connections made and signal p.c. boards attached with a total of $560-80$ stainless steel screws per gas manifold. Five twisted-pair 22 AWG nichrome heater-wire circuits [29] were installed on the outer surface of each CDT sector in the grooves between adjacent drift tubes, on the regions between the 6 aluminum sector ribs. Twisted pair heater wire was used instead of single wire to preclude interaction of the heater circuit and the magnetic field of the solenoid magnet generating unwanted forces on the CDT array. The twisted pair heater wire was first "tack-positioned" in place using thermo-setting glue and then potted with the 3X-Gray structural epoxy. The nominal resistance of each heater circuit was $220 \pm 5 \Omega$.

After each $30^{\circ}$ sector was completed, it was purged with dry nitrogen and 
then flushed with a mixture of argon-ethane $50 \%-50 \%$ gas bubbled through isopropyl alcohol at $5^{\circ} \mathrm{C}$. Each CDT sector was then fully tested using RABBIT readout electronics and a $\mu \mathrm{VAX}$ - II data acquisition system. The online program enabled rapid and simultaneous monitoring of all relevant operating parameters for every drift tube in the CDT sector being tested, such as singles counting rates, individual and summed pulse heights, TDC's, Z-distributions, wire participations/occupancies by ADC, TDC, etc. The data acquisition system could be triggered on single (and/or multiple) cosmic rays, $\mathrm{Fe}^{55}$ sources, and electronic charge injection signals. Because great efforts had been made to fully inspect and test all of the various components at every stage during the entire process of fabrication of the CDF Central Drift Tube array, no difficulties or surprises were encountered during the testing phase. There were no hot (or dead) wires, the counting rates, pulse height distributions and wire participations/occupancies were all very uniform for all CDT sectors. Fig. 10 shows the distribution of drift tube counting rates for 1,008 pairs of ganged tubes in the CDT array as measured with the $\mu \mathrm{VAX}$ - II data acquisition system.

After check-out, the CDT sectors were transported to Fermilab in individual foam-cushioned wooden boxes. There, each sector was bolted onto the CTC via the use of two precision NCM-machined 6061 T-6 aluminum sector mounting brackets, which attached to the rear portion of the CDT sector's HV pods. Each sector mounting bracket held 14 stainless steel press-fit dowel pins and 10-32 screws for the $7 \mathrm{HV}$ pods on each end of the sector, and four non-conducting 
molybdenum-disulphide filled plastic linear bearings [30]. Stainless steel 1/420 shoulder pins through the bearings attached the CDT sector to the CTC endplates at their outer periphery. The CDT array was rigidly attached at the east end of the CTC and freely attached on the west end to allow for axial motion due to differential thermal expansion/contraction of the CDT array relative to the CTC. Pairs of CDT $30^{\circ}$ sectors were electrically isolated from other pairs to minimize Eddy currents in the CDT array in the event of a quench of the CDF superconducting solenoid magnet.

The ADC pedestal width distribution for the entire CDT array (after installation in the CDF detector) using the CDF data acquisition system is shown in Fig. 11. The average pedestal width is $\sigma \sim 3 \mathrm{ADC}$ counts or $\sim 11.4 \mathrm{fC}$. The pulse height distribution for all 2,016 drift tubes from $\sqrt{ } \mathrm{s}=1.8 \mathrm{TeV} \dot{\mathrm{p} p}$ collisions is shown in Fig. 12. The average pulse height is $\sim 11.3 \mathrm{~K} \mathrm{ADC} \mathrm{counts,}$ or $43 \mathrm{pC}$ (into $50 \Omega$ ). The narrow peak in the pulse height distribution is due to single-streamer pulses, the secondary peak is due to double-streamer pulses and the tail is due in part multiple-streamer pulses and the geiger pulse component of the pulse height distribution. Typical drift tube hit occupancies of the CDT array for $\sqrt{ } \mathrm{s}=1.8 \mathrm{TeV} \overline{\mathrm{p}} \mathrm{p}$ events are $\sim 5 \%$, with an average number of hits per layer of tubes of $\left\langle\mathrm{N}_{\text {hit }}\right\rangle \simeq 35 \pm 23$.

\section{Conclusions}

We have designed, built, installed and operated a 2,016 wire Central Drift 
Tube array for use in the CDF experiment. The CDF Central Drift Tube array operates in the limited streamer mode, and obtains high-accuracy 3-dimensional $r-\phi-z$ information via charge division and drift time measurements at a radius of $1.4 \mathrm{~m}$, for tracking charged particles inside a $1.5 \mathrm{~T}$ magnetic field volume produced in the central region from $\sqrt{ } \mathrm{s}=1.8 \mathrm{TeV}$ p p collisions. The CDT array has successfully incorporated a novel, low-mass design for the precise registration and rigid support of drift tubes in the array using precision NCMmachined aluminum ribs and structural epoxy. The Central Drift Tube array performed well and operated reliably during the 1986-87 CDF data-taking run. Work is currently in progress to obtain the necessary calibration constants for the array. The calibration and operating performance of the CDT array in the CDF experiment will be discussed in a forthcoming paper [31].

\section{Acknowledgements}

Many people contributed directly and indirectly to the success of this project. The authors wish to thank all of the members of the University of Illinois' High Energy Physics Group for their help and advice throughout the course of the project. We wish to especially thank Mr. F.D. Cogswell for his supreme mechanical skills and tireless efforts throughout the entire project. Many of the crucial mechanical concepts and aspects of the CDT array, such as the use of the aluminum sector ribs, much of the tooling, jigs, techniques, etc. originated from him. We also wish to thank Mr. F. Witt of the University 
of Illinois Physics Department for his technical help and advice throughout the project. We also wish to explicitly thank the many people, who worked the countless long hours to bring this project to its successful, on-time completion. They are: F.D. Cogswell, T. Trumpinski, R. Spooner, M. Crouch, H.M. Blatz, M. Carey, R. Carey, M. Conlin, F. Damberger, G. Guglielmo, W.M. Harris, W.E. Johns, B.C. Jones, O. Krueger, B. Knobloch, A. Mertogul, L. Meyer, J.P. Raphael, A. Soni, M. Stoelinga, C. White and M. Zimmer. We also wish to especially thank Prof. L.E. Holloway for much technical help, and advice. We also wish to thank Profs. G. Ascoli, U. Kruse, T. O'Halloran, R.D. Sard, J.Thaler, Dr. I. Karliner, and also R. Downing, V.J. Simaitis, L. Peek, M.J. Pefalas, H. Scott, W. Whitten, and also D. Lesny, J. Wray, and D.A. Smith, T.K. Westhusing and also W. Testory, J. Adomaitas and D. Schoeps for their expert help and assistance throughout the project. Special thanks go to Profs. V.E. Barnes, A. Garfinkel and G. Durr of Purdue University for invaluable help in the exquisite fabrication and testing of all of the CDT array's 168 signal cable assemblies. Special thanks go to Dr. L.A. Errede of 3 M Research for much expert technical advice associated with various physical chemistry aspects of O-rings. The authors also wish to thank all of the CDF collaboration for their help and advice throughout the course of the project. Special thanks go to $\mathrm{M}$. Atac, for much helpful advice and information associated with many aspects of the operation of drift tubes in the limited streamer mode. We also wish to thank M. Anderson, A.R. Baden, A. Beretvas, J.P. Berge, C. Blocker, M. Bink- 
ley, J. Bofill, D. Brown, A. Byon, M. Campbell, T.J. Carroll, R. Cashmore, W. Chinowsky, J. Cooper, C. Day, T. Droege, J. Elias, G.W. Foster, J. Freeman, J. Grimson, S. Hahn, J. Huth, H. Jensen, R.W. Kadel, H. Kautzky, R.D. Kephart, S. Kuhlmann, A. Laasanen, W. Li, T. Liss, N.S. Lockyer, A. Mukherjee, C. Nelson, C. Newman-Holmes, L. Nodulman, J. O'Meara, A. Para, J. Patrick, J. Proudfoot, D. Quarrie, R. Schwitters, S. Segler, M.D. Shapiro, R. St.Denis, D. Theriot, A. Tollestrup, B. Troemel, K. Turner, R. Vidal, R. Wagner, J. Walsh and G.P. Yeh.

This work was supported in part by the U.S. Department of Energy, contract numbers DE-AC02-76ER01195 (UI) and DE-AC02-76CH03000 (FNAL), seed money from the University of Illinois Department of Physics, the University of Illinois College of Engineering, and a University of Illinois Arnold O. Beckman Research Award from the University of Illinois Research Board. One of us (S.E.) would also like to gratefully acknowledge the receipt of an Alfred $\mathbf{P}$. Sloan Research Fellowship Award.

\section{References}

[1] F. Bedeschi, et al, The Design and Construction of the CDF Central Tracking Chamber, submitted to Nuclear Instruments and Methods, 1987.

[2] F. Snider, et al, The CDF Vertex Time Projection Chamber Sys- 
tem, submitted to Nuclear Instruments and Methods, 1987.

[3] S. Bhadra, et al, The CDF Central Drift Tube Level-I Trigger Electronics, to be submitted to Nuclear Instruments and Methods, 1987.

[4] G. Ascoli, et al., The CDF Central Muon Detector, submitted to Nuclear Instruments and Methods, 1987; and G. Ascoli, et al., The CDF Central Muon Level-I Trigger Electronics, submitted to Nuclear Instruments and Methods, 1987.

[5] L. Balka, et al., The CDF Central Electromagnetic Calorimeter, submitted to Nuclear Instruments and Methods, 1987.

[6] D. A. Smith, Monte Carlo Simulation Studies of $\sqrt{ } \mathbf{s}=1.8 \mathrm{TeV} \overline{\mathbf{p}} \mathbf{p}$ Collisions and the Design of the CDF Central Drift Tube Array, University of Illinois Preliminary Exam Ph. D. Paper, 1985 (unpublished).

[7] H. M. Blatz, A Systematic Study of the Properties of Structural Epoxies for Use in the CDF Central Drift Tube System, University of Illinois Independent Study Report, 1986 (unpublished).

[8] S. Bhadra, et al., High Accuracy Charge Division from Drift Tubes Operated in the Limited Streamer Mode, to be submitted to Nuclear Instruments and Methods, 1987.

[9] The 304 stainless steel tubes used in the CDF Central Drift Tube array were manufactured by Uniform Tubes, Inc., Seventh Ave., Collegville, PA, 
19426 USA.

[10] Drift tubes were made with four principle wire support locations. The eight distinct wire support locations in the CDT array were achieved simply by rotating one-half of the tubes made with the four principle wire support locations by $180^{\circ}$ (about a perpendicular to the tube axis) during fabrication of $30^{\circ} \mathrm{CDT}$ sectors.

[11] The large diameter PRP-568-011 Viton 9041-75 O-rings used for gas sealing on the CDT array were obtained from Parco O-Ring Division, Inc., 2150 Parco Ave., Ontario, CA, 91761 USA.

[12] The $0.033 \mu \mathrm{F}$ Metfilm-E 4KV Type 430P-333-X9-40 HV blocking capacitors used in the CDT array were manufactured by the Sprague Electronics Co., Park Ridge, IL, 60068 USA.

[13] The miniature $\mathrm{HV}$ coaxial cable and miniature $\mathrm{HV}$ connectors used in the CDF Central Drift Tube array were manufactured by Reynolds Industries, Inc. 5005 McConnell Ave., Los Angeles, CA, 90066 USA.

[14] The miniature 60-pin surface mount p.c. board connectors used in the CDF Central Drift Tube array were manufactured by the DuPont Co., Berg Elec. Div., New Cumberland, PA, 17070 USA.

[15] The miniature 26-conductor coaxial signal cables used in the CDF Central Drift Tube array were manufactured by New England Wire Co., 365 Main St., Lisbon, NH, USA. 
[16] The ceramic saw blades used to cut the stainless steel drift tubes were manufactured by the Valley Forge Grinding Wheel Company, $101 \mathrm{~W}$. Second St., Bridgeport, PA, 19403 USA.

[17] The air-drying system used in the drift tube cleaning process was obtained from Enpro, Inc., 121 S. Lombard Rd., Addison, IL, 60101 USA. The Enpro air drying system operated at an air temperature equivalent of $-85^{\circ} \mathrm{C}$, in terms of its water vapor content.

[18] Kapton is a trademark for a polyamide film manufactured by DuPont de Nemours, E.I. Co., Wilmington, DE, 19868 USA.

[19] The Stablohm-800 $50 \mu \mathrm{m}$ diameter, stress-relieved, ultra-finished, nonmagnetic anode wire used in the CDF Central Drift Tube array was obtained from California Fine Wire, Inc., 338 S. Fourth St., P.O. Box 446, Grover City, CA, 93433 USA.

[20] S. Bhadra, et al., A Wire Tension Measurement System Used in the Fabrication of the CDF Central Drift Tube array, submitted to Nuclear Instruments and Methods, 1987.

[21] The $\mathrm{Fe}^{35}$ source material used in the CDF Central Drift Tube array was obtained from Isotope Products Laboratories, 1800 N. Keystone St., Burbank, CA, 91504 USA.

[22] The Dexter Hysol 3X Gray structural epoxy used in CDT Sector fabrication was obtained from the Electro Insulation Corp., 2535 Clearbrook Dr., 
Arlington Heights, IL, 60005 USA.

[23] The cartridge brass tubing used for the Central Drift Tube array's shotgun shellend assemblies was obtained from H \& H Tubes, 400 Town Center Rd., Southfield, MI, 48037 USA.

[24] The RTV-11 used for potting the brass shotgun shell end assemblies was obtained from the Electro Insulation Corp., 2535 Clearbrook Dr. Arlington Heights, IL, 60005 USA.

[25] The conductive rubber used to make the zero-stress electrical connection between the drift tubes and the brass shotgun shell end assemblies was obtained from Chomerics, Inc., 77 Dragon Ct., Woburn, MA, 01801 USA.

[26] The small "quad" 003 Viton 4003-514AD O-rings used for gas sealing in the CDT array's gas manifolds were obtained from Minnesota Rubber, 3630 Wooddale Ave., Minneapolis, MN, 55416 USA.

[27] The LX06030A solid-state pressure transducers used in the CDT array were obtained from SenSym, Inc., 1255 Reamwood Ave., Sunnyvale, CA, 94809 USA.

[28] The GB32J2 and GB34P2 thermistor temperature sensors used in the CDT array were obtained from Fenwal Electronics, Inc., 63 Fountain St., Framingham, MA, 01701 USA.

[29] The 22 AWG nichrome heater wire used in the CDF Central Drift Tube 
array's heater circuitry was obtained from the Pelican Wire Company, Inc., 6266 Taylor Rd., Naples, FL, 33942 USA.

[30] The LMN-1 molybdenum disulphide-filled plastic "no-lube" linear bearings used in the CDT sector mounting brackets were obtained from W. M. Berg, Inc., 499 Ocean Ave., East Rockaway, Long Island, NY, 11518 USA.

[31] S. Bhadra, et al, Calibration and Performance of the CDF Central Drift Tube array, in preparation for submission to Nuclear Instruments and Methods, 1987. 


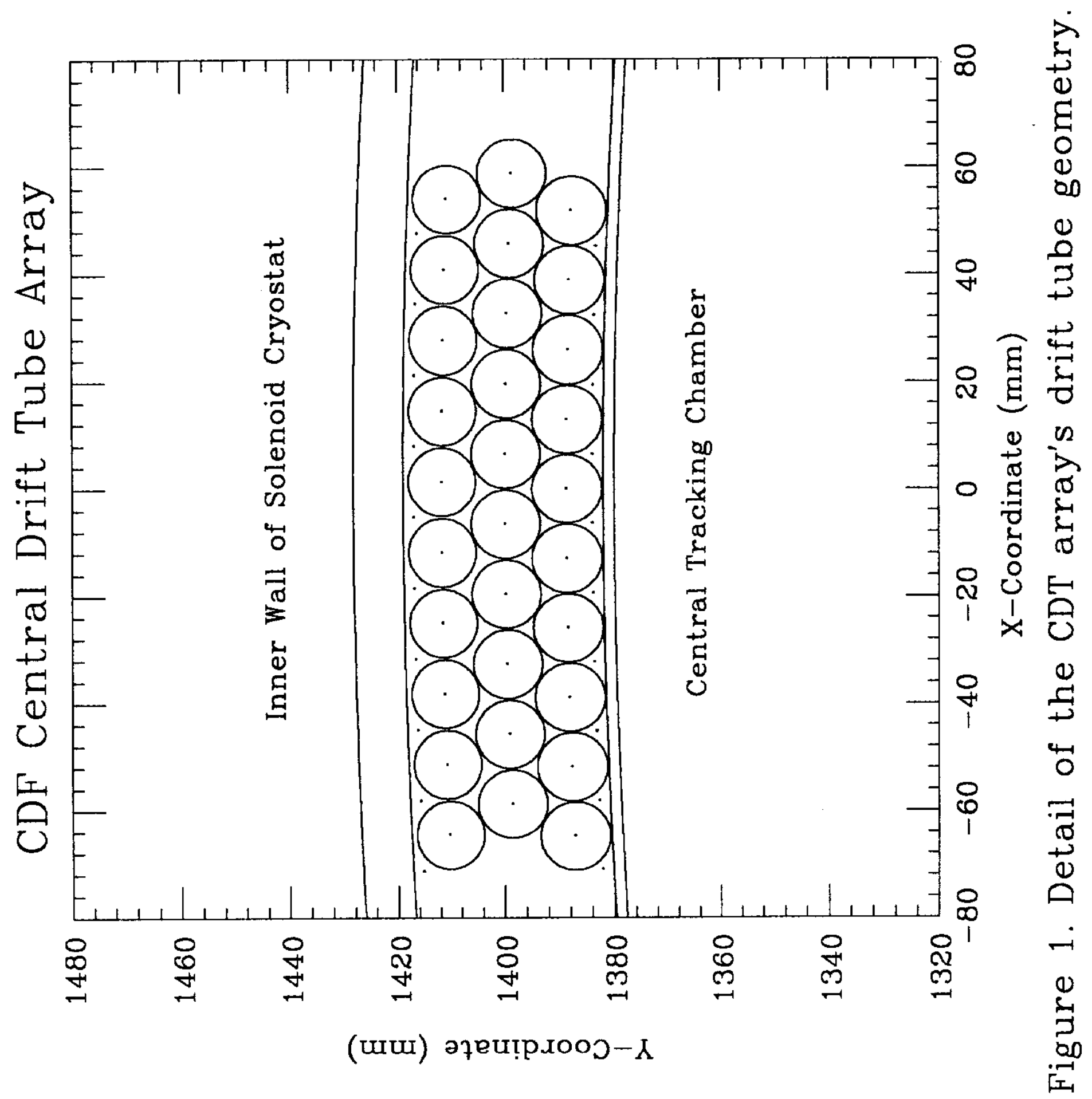




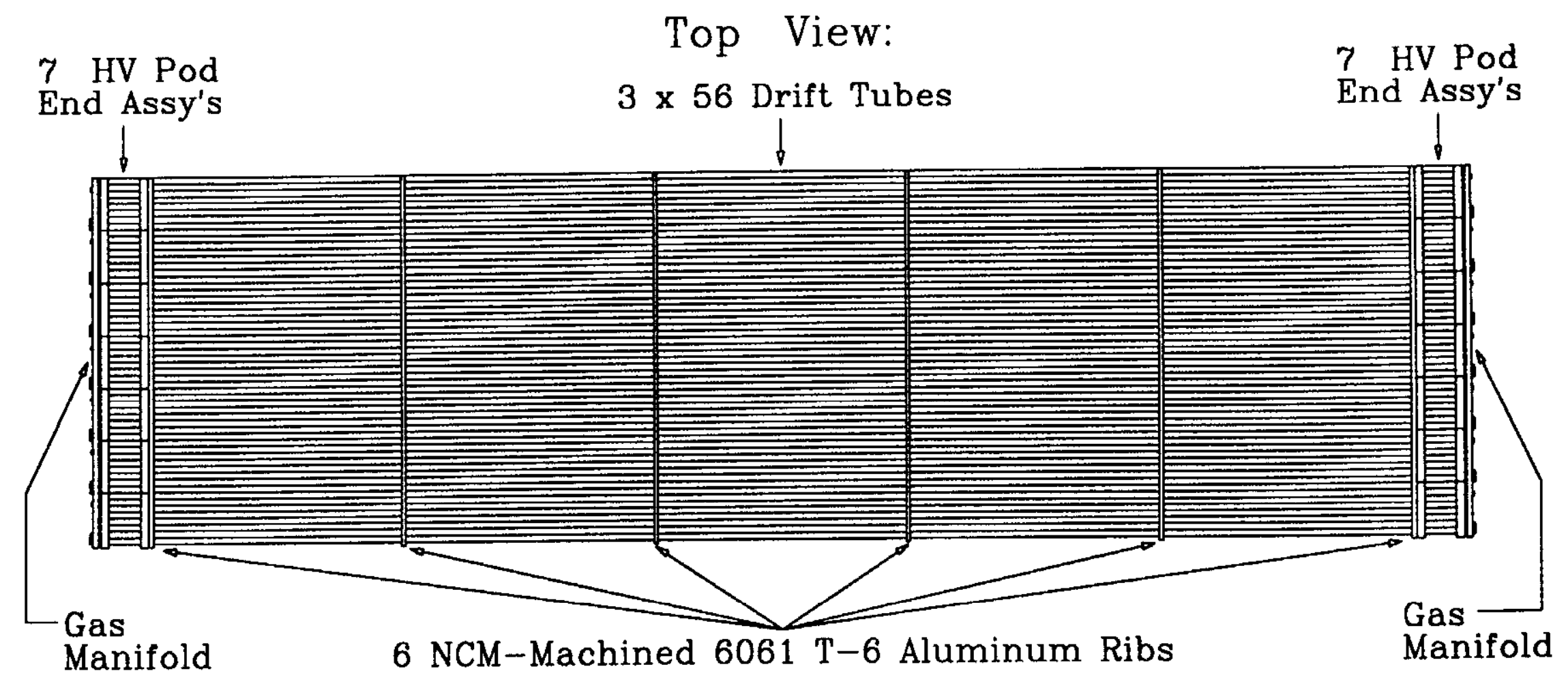

Side View:

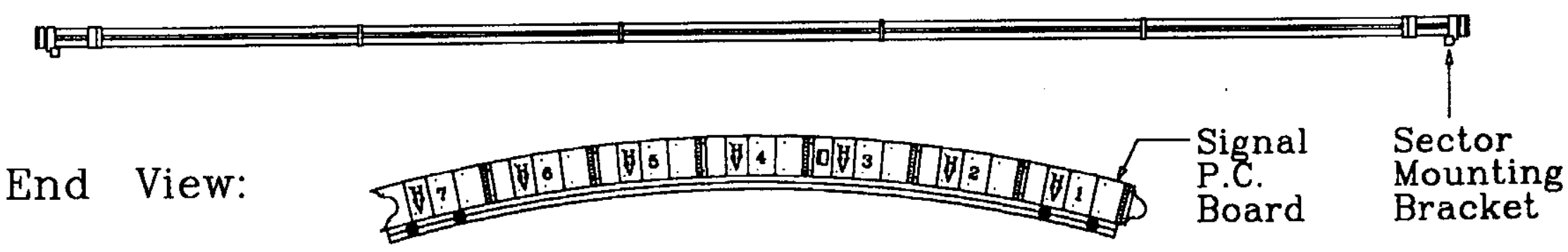

Figure 2. CDF Central Drift Tube $30^{\circ}$ Sector. 


\section{CDF Central Drift Tube Delrin Wire Support}

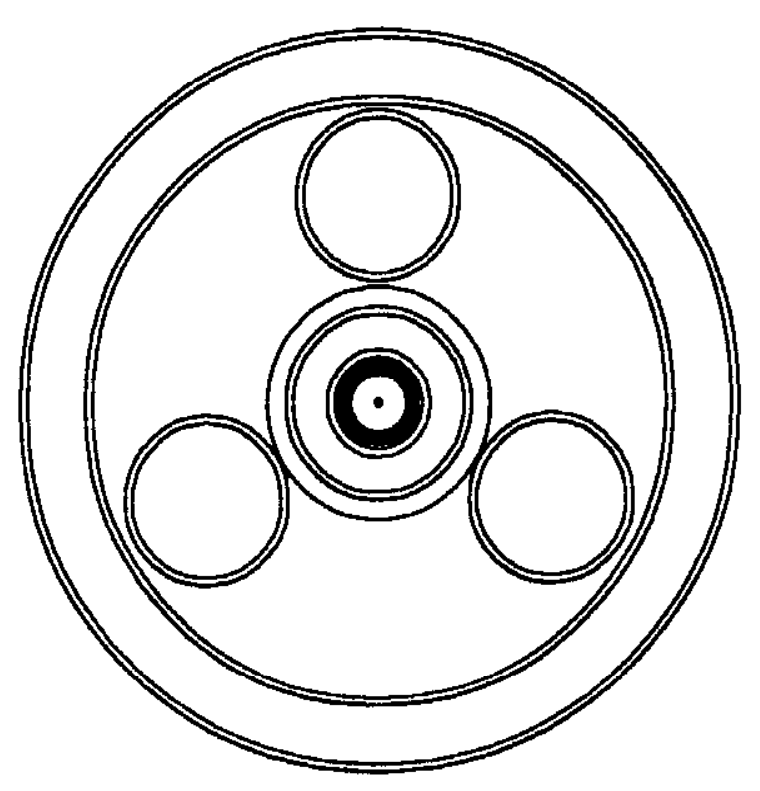

Front View

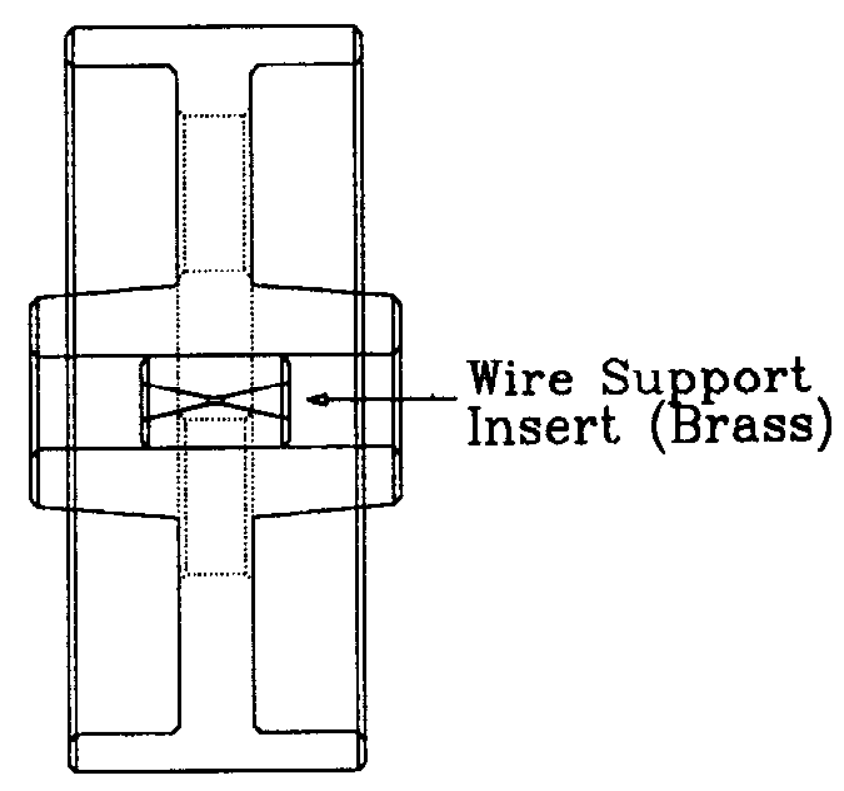

Side View

Figure 3. Detail of a drift tube delrin wire support. 
CDF Central Drift Tube Delrin Endpin/Feedthru

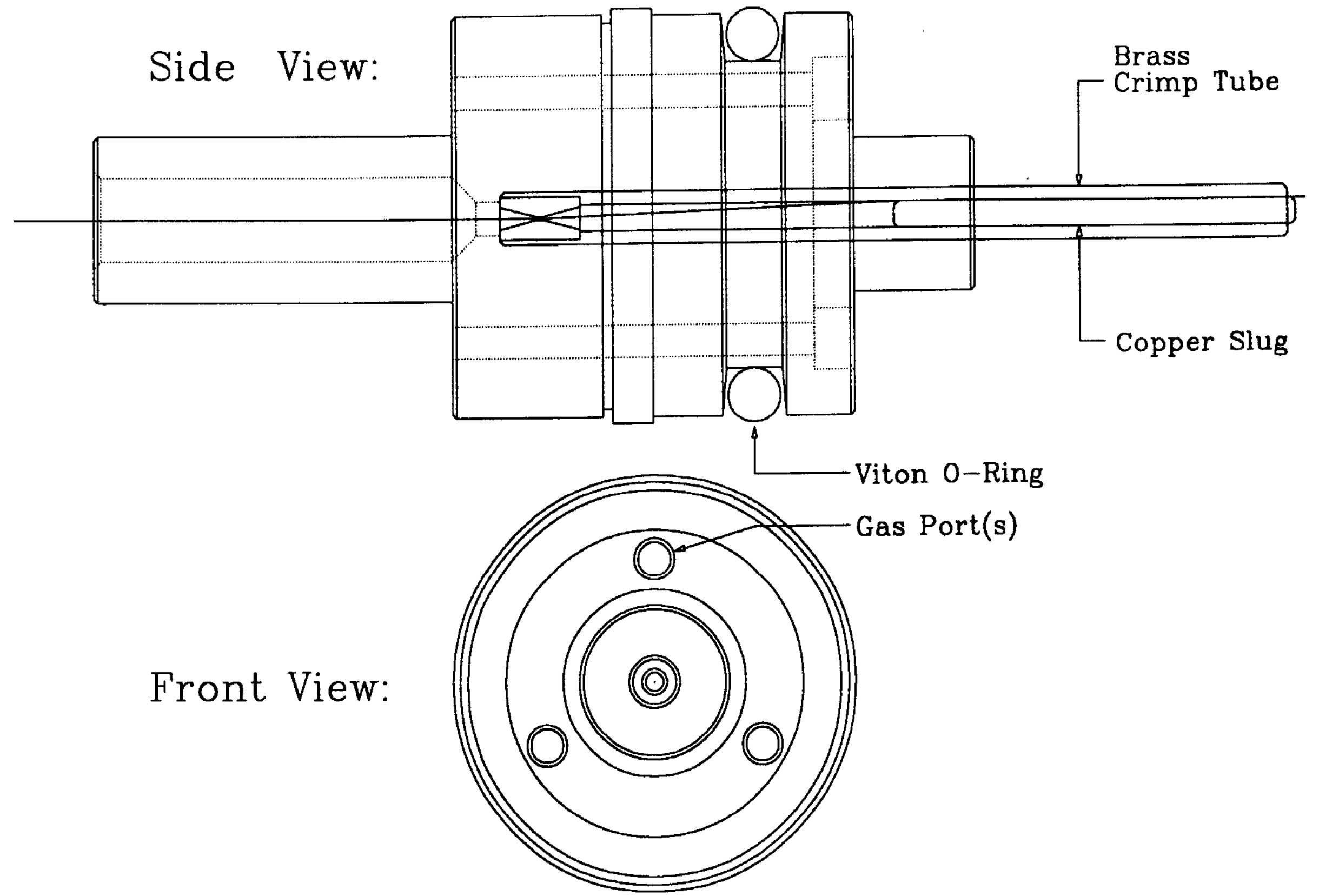

Figure 4. Detail of a drift tube delrin endpin/feedthru assembly. 
CDF Central Drift Tube Shotgun Shell End Assembly

Front View:

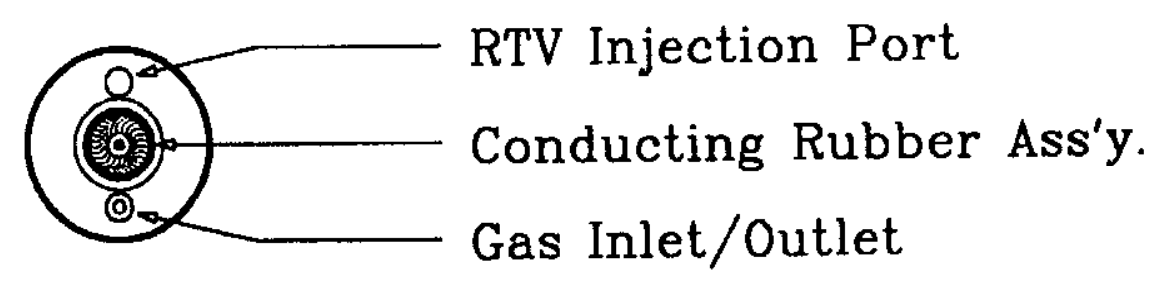

Side View:

Brass Shotgun

Shell

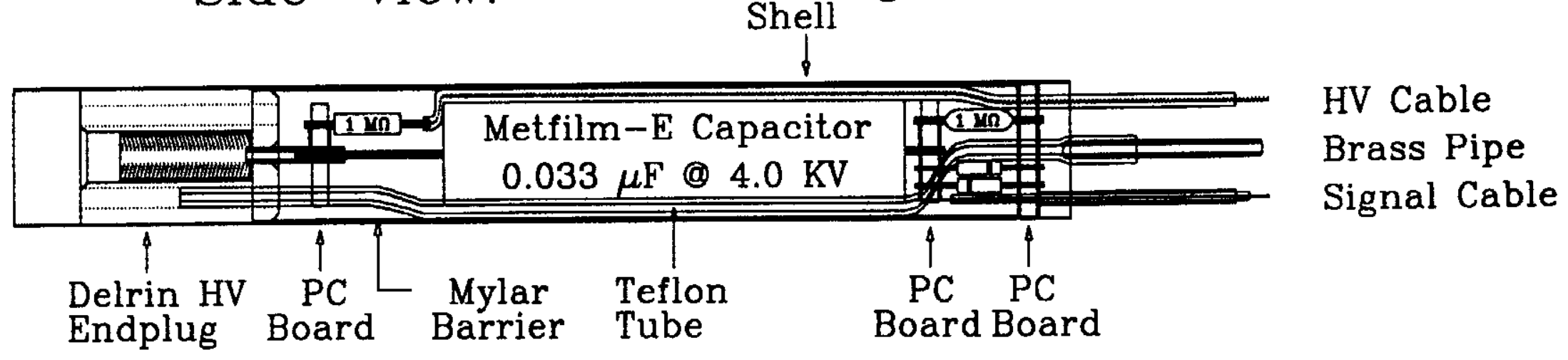

Rear View:

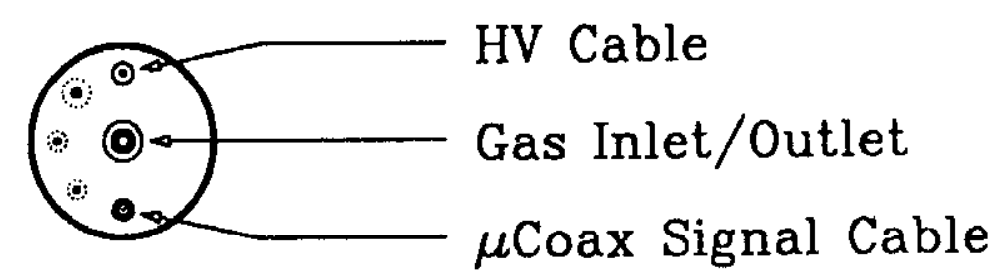

Figure 5. Detail of a drift tube brass shotgun shell end assembly. 


\section{CDF Central Drift Tube HV Pod End-Assembly}

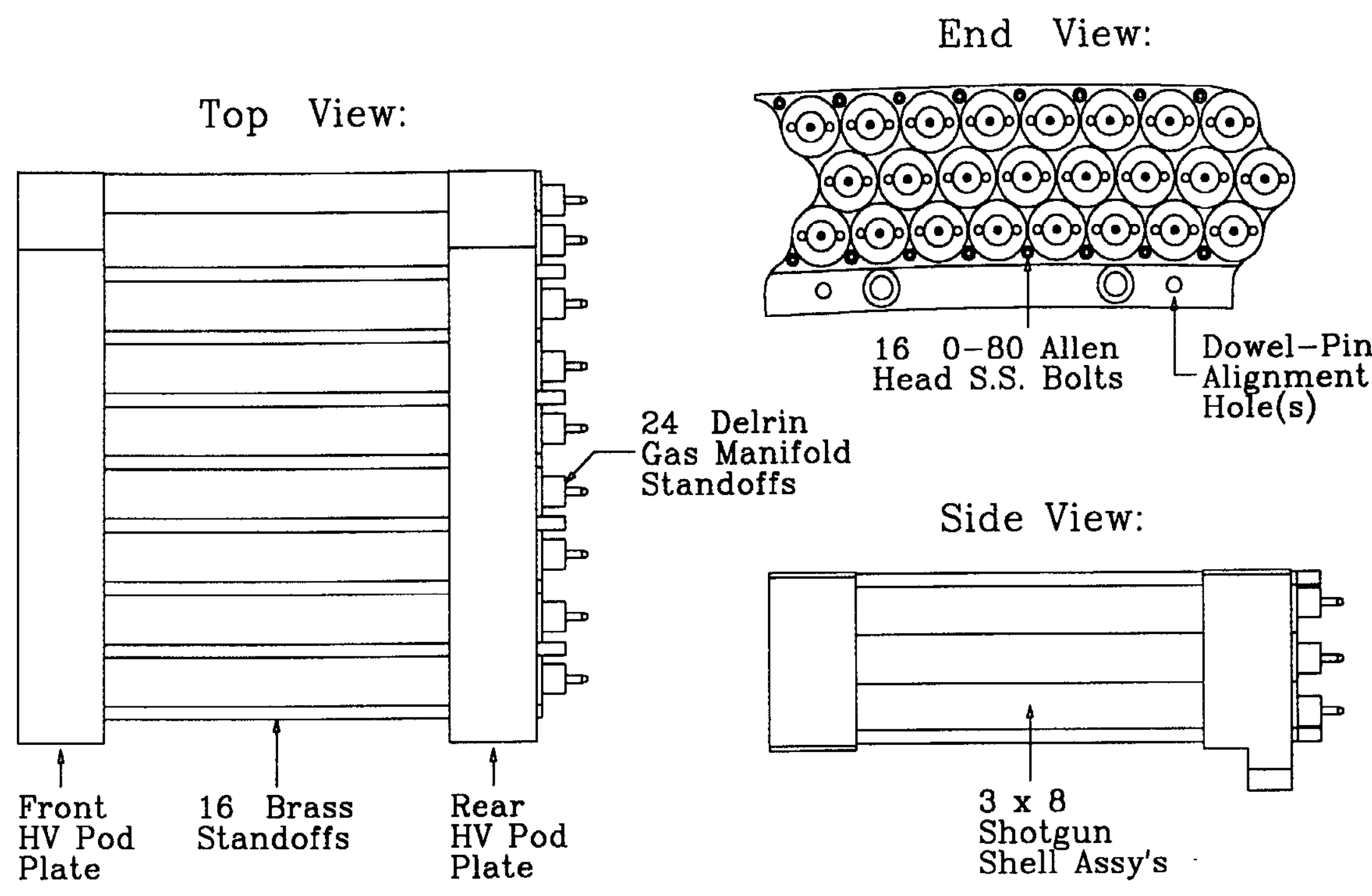

Figure 6. Detail of a Central Drift Tube HV Pod End-Assembly. 


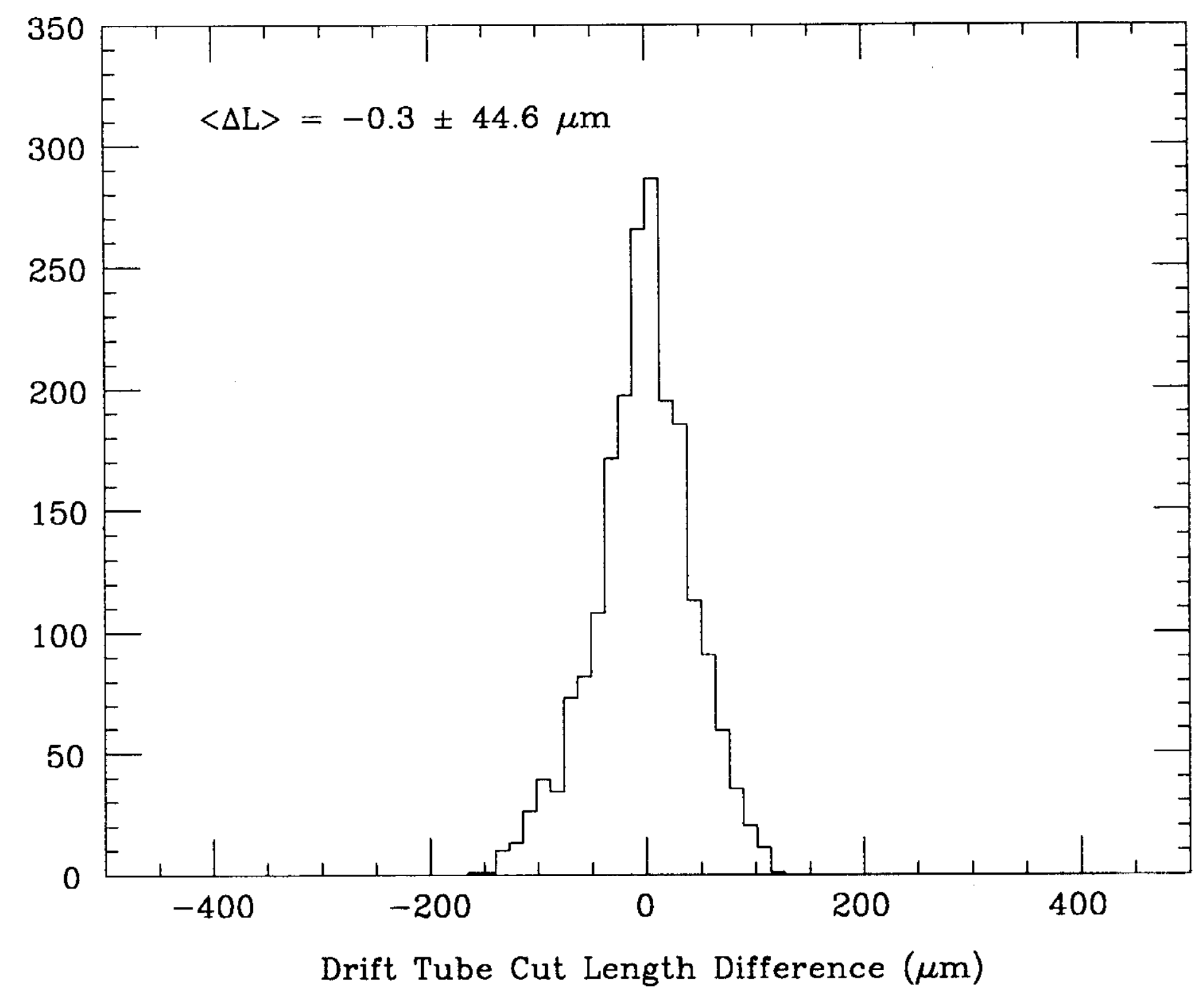

Figure 7. Deviation of measured drift tube lengths from design length. 


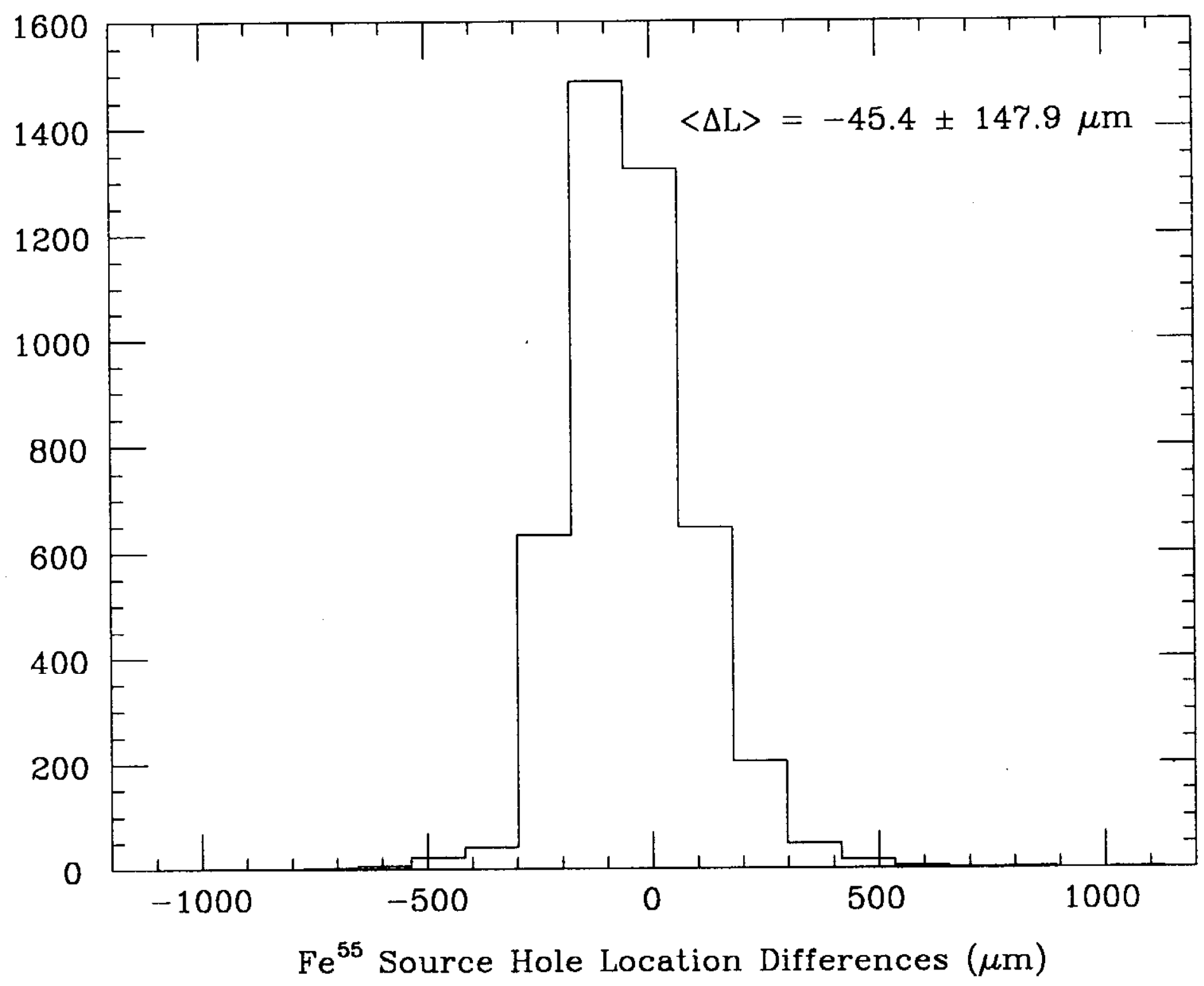

Figure 8. Deviation of drift tube $\mathrm{Fe}^{55}$ source hole locations from design. 


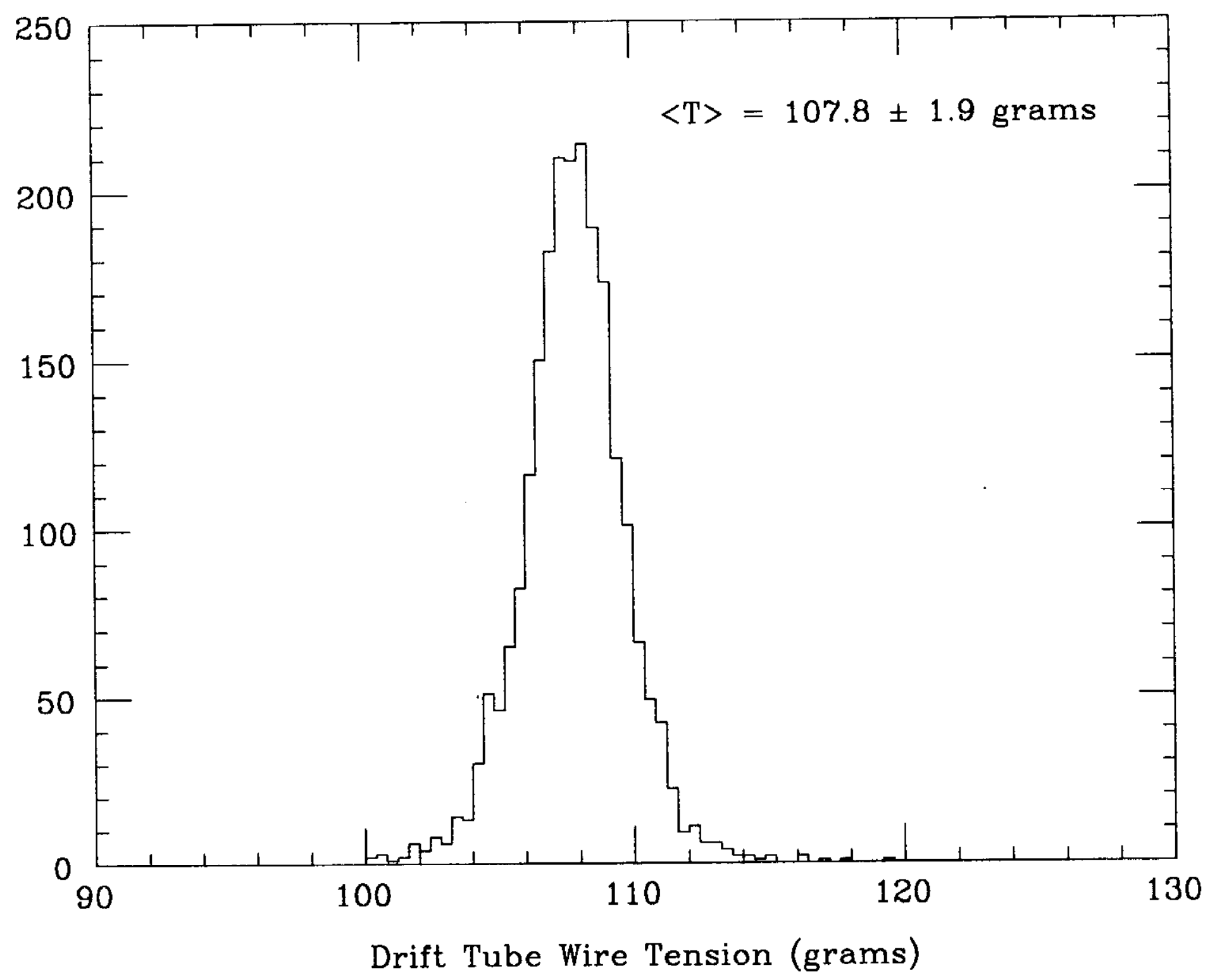

Figure 9. Wire tensions for 2,226 drift tubes. 


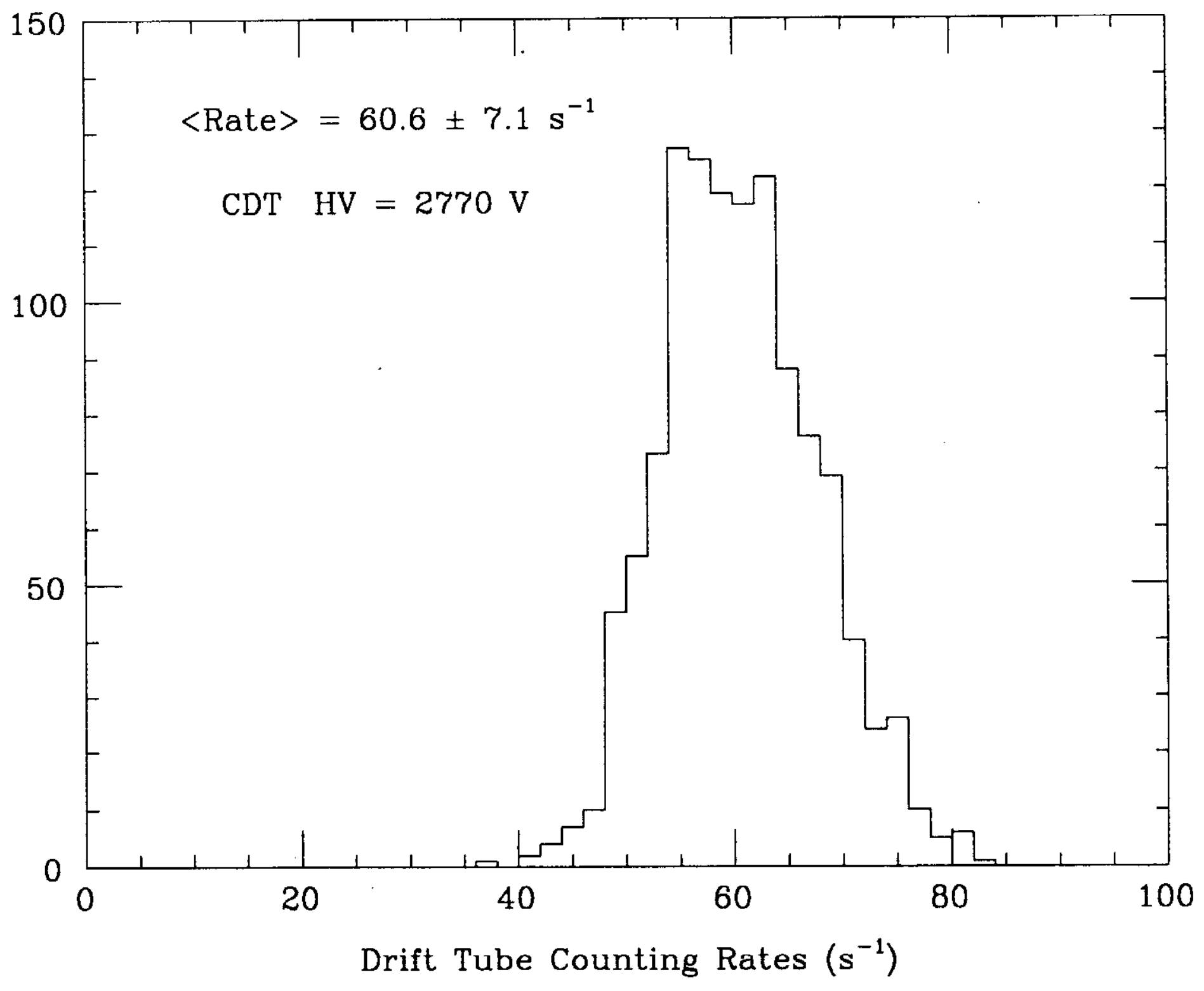

Figure 10. Counting rates for 1,008 pairs of ganged drift tubes. 


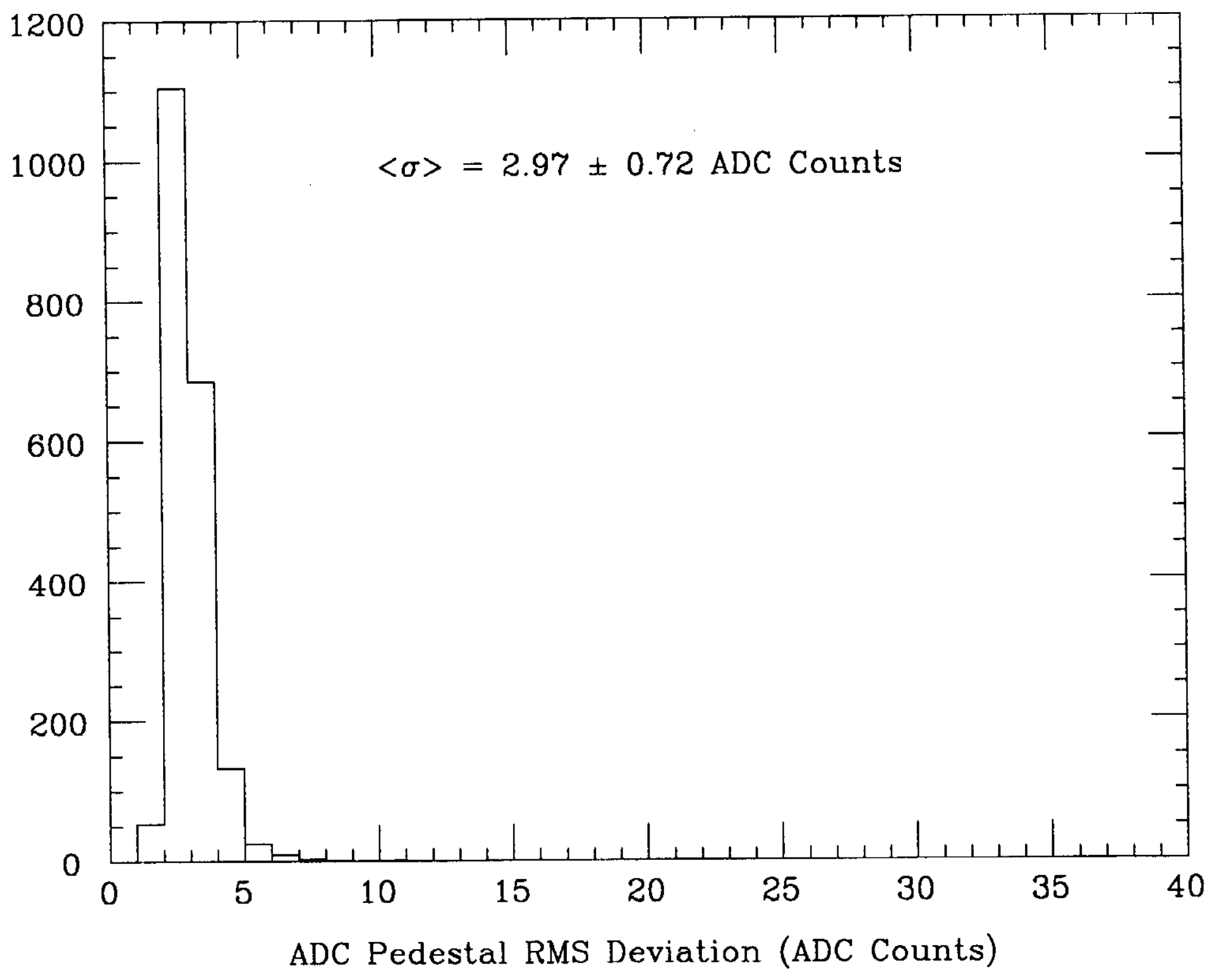

Figure 11. Pedestal Widths for 2,016 ADC channels. 


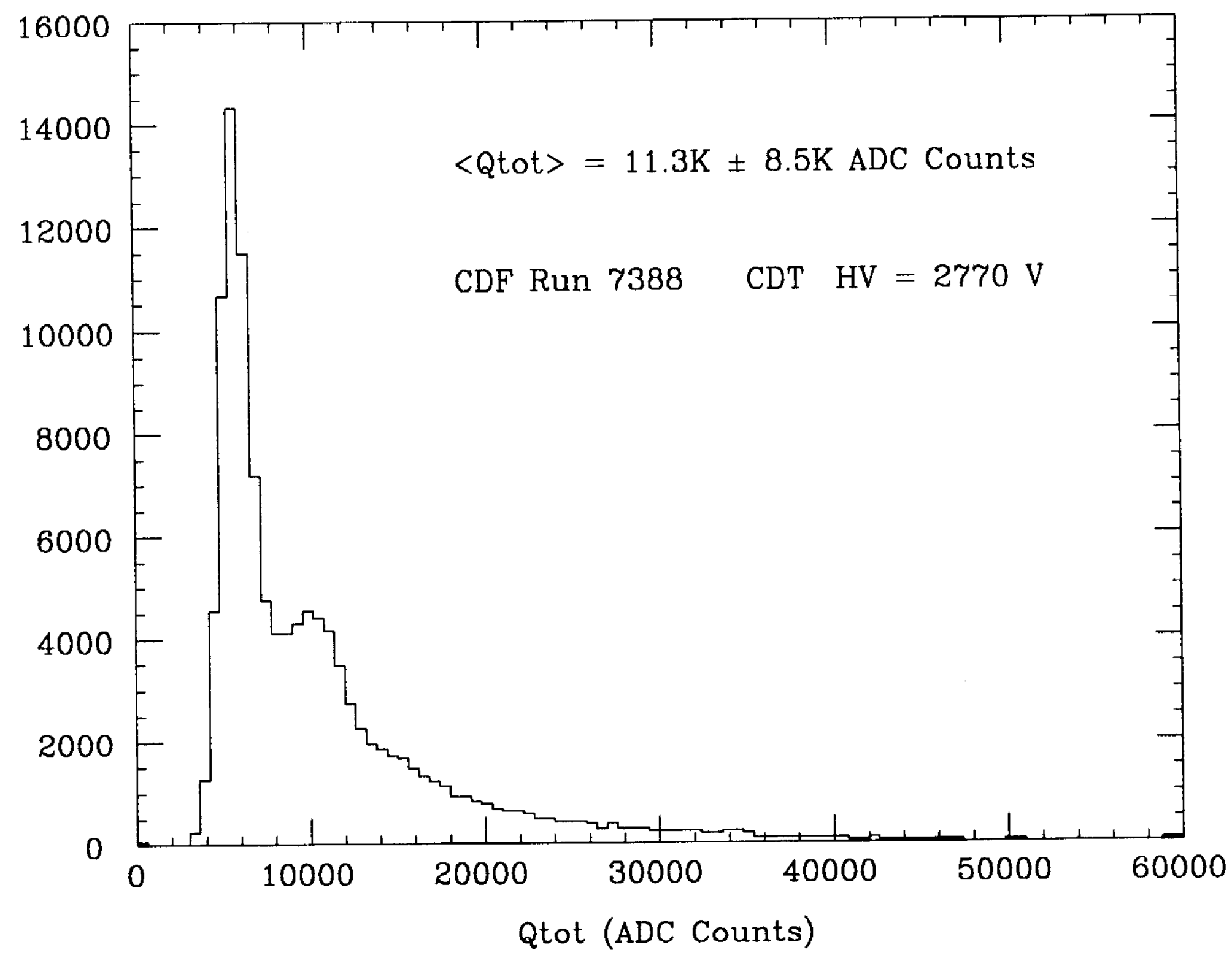

Figure 12. Pulse Height Distribution for 2,016 drift tubes. 